\title{
Surgical repair of Stanford type A aortic dissection in elderly patients: a contemporary systematic review and meta-analysis
}

\author{
Vito D. Bruno, Pierpaolo Chivasso, Gustavo Guida, Hunaid A. Vohra \\ Cardiac Surgery Department, Bristol Heart Institute, Bristol, UK \\ Correspondence to: Vito D. Bruno, MD, PhD. Cardiac Surgery Department, Bristol Heart Institute, Upper Maudlin Street, BS2 8HW, Bristol, UK. \\ Email: Vito.D.Bruno@bristol.ac.uk.
}

\begin{abstract}
Background: The results of surgical treatment of type A aortic dissection (AAD) in the elderly are controversial and aggravated by a higher operative mortality rate. The studies published in this subset of patients are mainly retrospective analyses or small samples from international registries. We sought to investigate this topic by conducting a contemporary meta-analysis of the most recent observational studies.

Methods: A systematic literature search was conducted for any study published in the last five years on aortic dissection treated surgically in patients 70 years and older. A pooled risk-ratio meta-analysis has been conducted three main post-operative outcomes: short-term mortality, stroke and acute kidney injury.

Results: A total of 11 retrospective observational studies have been included in the quantitative metaanalysis. Pooled meta-analysis showed an increased risk of short term mortality for the elderly population [relative risk $(\mathrm{RR})=2.25 ; 95 \% \mathrm{CI}, 1.79-2.83 ; I^{2}=0 \% ; \mathrm{P}<0.0001$ ], and this has been confirmed in a sub-analysis of patients 80 years and older. The risk of having stroke $\left(\mathrm{RR}=1.15 ; 95 \% \mathrm{CI}, 0.89-1.5 ; I^{2}=0 \% ; \mathrm{P}=0.28\right)$ and acute kidney injury $\left(\mathrm{RR}=0.79 ; 95 \% \mathrm{CI}, 0.5-1.25, I^{2}=14 \%, \mathrm{P}=0.31\right)$ after surgery were comparable to the younger cohort of patients.

Conclusions: Although affected by an increased risk of short-term mortality in the elderly, surgical repair remains the treatment of choice for $\mathrm{AAD}$. The main post-operative outcomes are comparable to younger patients and the mid-term survival rates are acceptable.
\end{abstract}

Keywords: Aortic dissection; elderly; meta-analysis; systematic review

Submitted Apr 05, 2016. Accepted for publication Jun 02, 2016.

doi: $10.21037 /$ acs.2016.06.03

View this article at: http://dx.doi.org/10.21037/acs.2016.06.03

\section{Introduction}

Despite its relative rarity, type A aortic dissection (AAD) remains a serious and often lethal disease, with in-hospital mortality ranging between 16 and $83 \%$ (1). Older age represents a risk factor for surgical treatment (2) and previously published data from The International Registry of Acute Aortic Dissection (IRAD) found age $\geq 70$ years as a significant predictor of in-hospital surgical mortality (3). In contrast, a meta-analysis published in 2011 (4) and focused on octogenarian patients showed satisfactory immediate survival rate after surgery and suggested a confident approach toward emergency repair in this complex subset of patients. Enhancements in diagnostics, surgical techniques and post-operative care have certainly improved over the last years and more recent registry reports and retrospective analysis have revealed very good results after AAD surgical repair in the aged population, supporting surgical intervention even in very elderly patients $(5,6)$. We investigated the current evidence on the surgical outcome of AAD repair in elderly patients by conducting a contemporary systematic review and meta-analysis of the latest published studies on this topic.

\section{Methods}

\section{Search strategy and study selection}

A systematic literature search was conducted through PubMed for any study published in the last five years on 
aortic dissection; an additional search was performed in the same period of time aiming at studies evaluating the surgical outcomes of type A aortic dissection repair in the elderly. Searching phrases used were: ["Aortic Dissection" (all fields)] and a combination of this term with the subsequent terms: [elderly (all fields)], \{elderly [MeSH term]\}, [aged (all fields)], \{aged [MeSH term]\}, [aging (all fields)], \{aging [MeSH term]\}, [geriatrics (all fields)] and \{geriatrics $[\mathrm{MeSH}$ term]\}. To further identify the papers of interest, a cross check with the same searching criteria was conducted on Scopus. The five year time period was used in consideration of a previous meta-analysis published in 2011 on a similar topic of interest (4). Two reviewers (V.D.B. and P.C.) independently assessed the online databases (last access on the $3^{\text {rd }}$ May 2016), screening titles and abstracts. The full-text articles were then obtained for all potentially eligible articles that clearly met the inclusion criteria and were reviewed separately if either reviewer considered the manuscript as being eligible. Any disagreements were resolved by consensus.

\section{Eligibility criteria}

Case reports, editorials, reviews and meta-analysis were excluded. Non-clinical or post-mortem reports were also excluded. The selection inclusion criteria were: (I) articles addressing acute type A aortic dissection; (II) including age groups specifications (or obtainable from presented data); (III) reporting short term mortality and/or post-operative stroke rate and/or post-operative acute kidney injury. All publications were limited to human subjects and written in English.

\section{Data extraction}

All data were independently extracted from the studies by two investigators (V.D.B. and P.C.). The final results were reviewed by the senior reviewer (H.V.). Our main outcomes of interest were short-term post-operative mortality, postoperative stroke and acute post-operative kidney injury (AKI). Data on those main outcomes were retrieved from the original articles. Short-term mortality was defined as in-hospital or 30-days post-operative mortality. In cases where both timing was available, only the in-hospital mortality was used. Stroke was defined as permanent postoperative neurological events as stated by the papers. Acute kidney injury was only considered if requiring temporary or permanent hemofiltration, according to the article

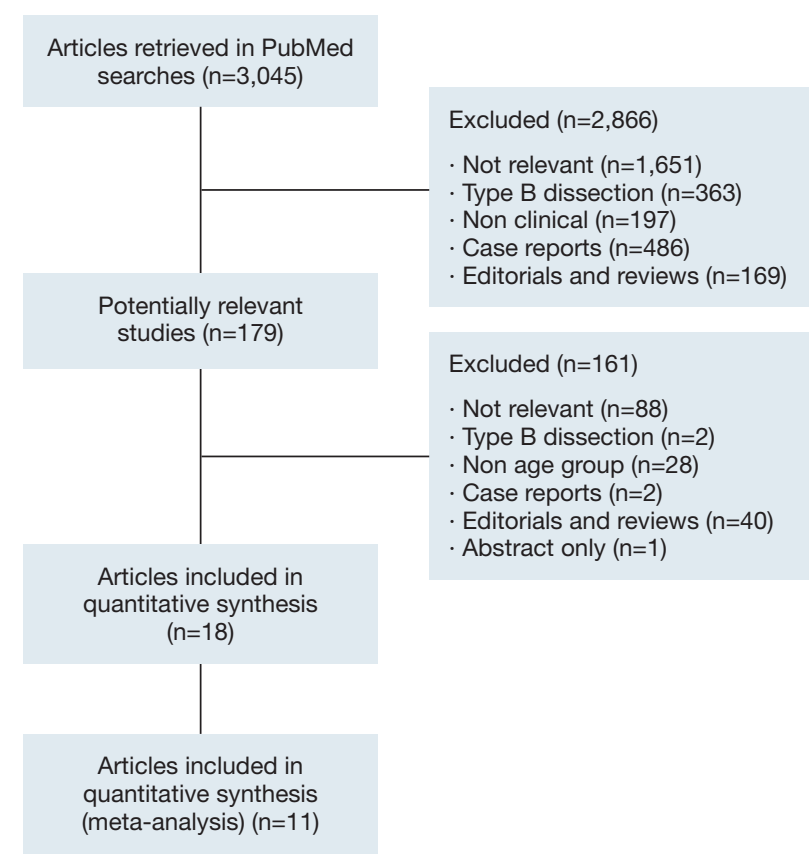

Figure 1 PRISMA diagram describing the search results. PRISMA, Preferred Reporting Items for Systematic Reviews and Meta-Analyses.

description. The definition of 'elderly' varied among the studies: therefore, we included in the meta-analysis only the studies involving patients with age $\geq 70$ years in the study population and conducted a sub-analysis on octogenarians only. Figure 1 describes the research protocol and selection process.

\section{Statistical analysis}

Study characteristics are presented as raw values and percentages. The dichotomous data are expressed as pooled proportion and $95 \%$ confidence interval. Risk ratio (RR) meta-analysis was conducted using inverse variance weighting method of individual log hazard ratios. Forest plots were used to describe the results reporting RR and 95\% confidence intervals (CI). The heterogeneity between studies was estimated using $\chi^{2}$-based $\mathrm{Q}$ statistics and $\mathrm{I}^{2}$ test. Statistical significance was set at $\mathrm{P}<0.05$. Statistical analysis was conducted with $\mathrm{R}$ statistical software (R Foundation for Statistical Computing, Vienna, Austria - package meta).

\section{Results}

A total of 3,045 studies were found on online databases. 


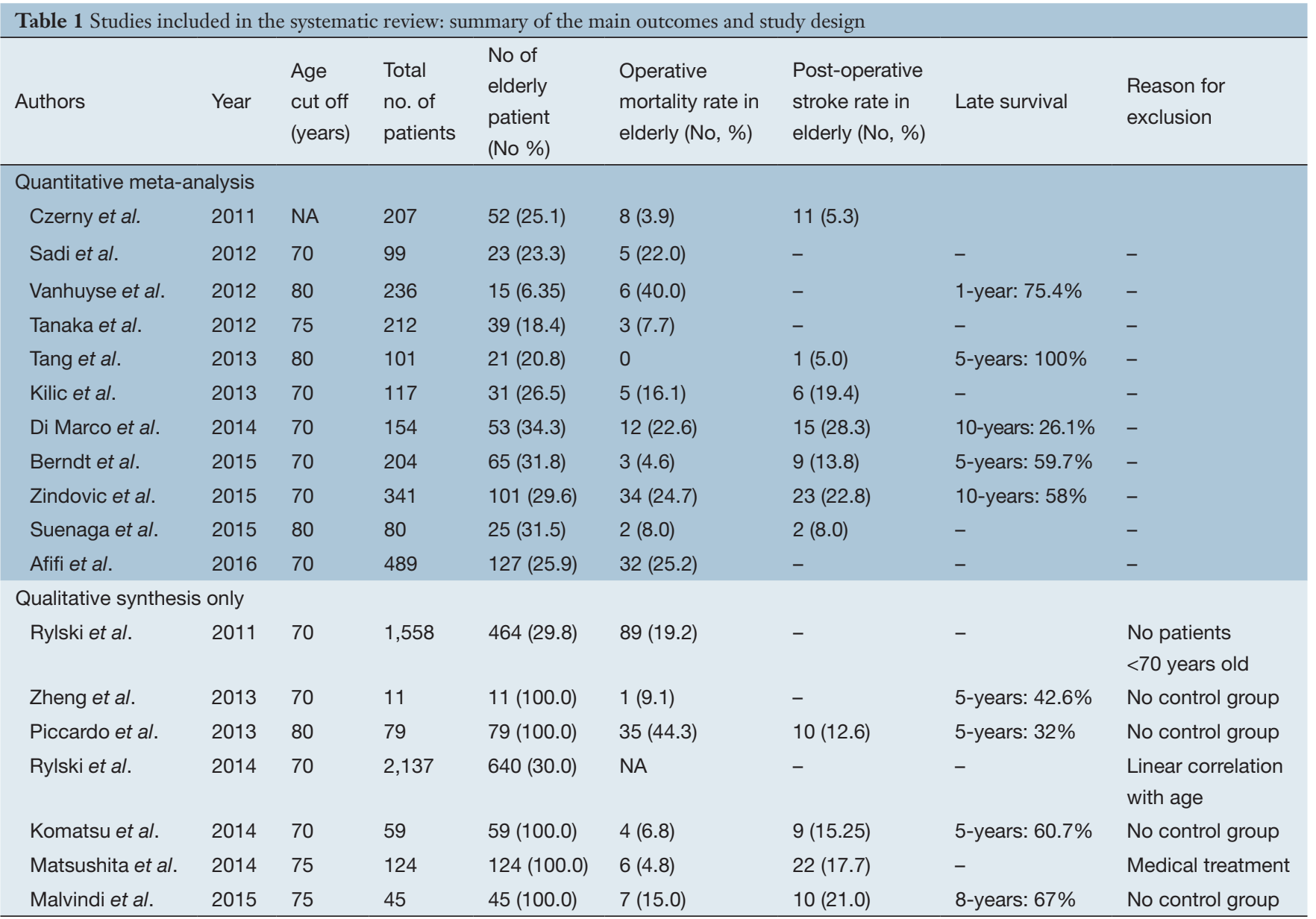

After initial screening, 179 studies were considered potentially relevant. A further screening of abstracts and full texts was conducted and a final sample of 18 studies considered relevant to our review (1,2,5,7-20). Of these, seven were excluded from the quantitative meta-analysis for different reasons. Table 1 describes the included and excluded studies, their main characteristics and the reason for exclusion from quantitative analysis. Four papers did not have a young control group and one paper considered medical treatment only. Two important papers from the German Registry for acute aortic dissection type A (GERAADA) have addressed the topic of surgical treatment in elderly patients $(5,18)$. We did not include them in the meta-analysis because one did not have a younger cohort ( $<70$ years old) and one did not report our main outcomes in a specific group of age, but analyzed a linear correlation between outcome and age. One paper (7) had no specific age cut-off, but reported mortality and stroke rate for different age groups. This was included in the meta-analysis and in this case we decided to consider 70 years old as cutoff for elderly group and the mortality and stroke rates were derived accordingly.

The intra-operative characteristics for the elderly patients are described in Table 2: these data were obtainable only from 11 studies. The mean cardiopulmonary bypass time and aortic cross clamp time ranged from 150 to 291.6 minutes and 70 to 191.3 minutes respectively. The deep hypothermic circulatory arrest time ranged from 18 to 99.7 minutes.

\section{Short term mortality}

Short term mortality was defined as in-hospital or 30-day mortality depending on the specific definition of each study and was reported in all eleven studies, accounting for a total of 552 elderly patients over an overall population of 2,180 


\begin{tabular}{|c|c|c|c|c|c|c|c|}
\hline Authors & $\begin{array}{l}\text { CPB time } \\
\text { (min) }\end{array}$ & $\begin{array}{l}\text { ACC time } \\
(\mathrm{min})\end{array}$ & $\begin{array}{l}\text { DHCA, n } \\
(\%)\end{array}$ & $\begin{array}{l}\text { DHCA time } \\
(\min )\end{array}$ & $\begin{array}{l}\text { Associated aortic } \\
\text { root replacement } \\
(n, \%)\end{array}$ & $\begin{array}{l}\text { Associated hemi/ } \\
\text { total aortic arch } \\
\text { replacement }(n, \%)\end{array}$ & $\begin{array}{l}\text { Associated } \\
\text { aortic valve } \\
\text { procedure }(n, \%)\end{array}$ \\
\hline Vanhuyse et al. & $194 \pm 59.3$ & $99 \pm 32.2$ & $11(73.3)$ & NA & $2(13.3)$ & $2(13.3)$ & NA \\
\hline Tang et al. & $186 \pm 48$ & $103 \pm 49$ & NA & $20 \pm 7$ & $23(28.0)$ & $53(66.0)$ & $23(28.0)$ \\
\hline Kilic et al. & $154.3 \pm 49.9$ & $77.6 \pm 49$ & NA & $22.6 \pm 13.7$ & 4 (12.9) & 4 (12.9) & $11(35.5)$ \\
\hline Zindovic et al. & $\begin{array}{l}201 \\
(182 \pm 243)\end{array}$ & 98 [72-141] & $29(30.9)$ & 18 [12-28] & $17(18.1)$ & 19 (20.2) & 17 (18.1) \\
\hline Suenaga et al. & $150 \pm 32$ & $70 \pm 15$ & NA & $30 \pm 4.9$ & NA & NA & NA \\
\hline Zheng et al. & $151 \pm 33.5$ & $68.5 \pm 41.4$ & $11(100.0)$ & $30.3 \pm 12.9$ & NA & $11(100.0)$ & NA \\
\hline Malvindi et al. & $153 \pm 73$ & $87 \pm 30$ & $22(49.0)$ & NA & $3(7.0)$ & $12(27.0)$ & $30(67.0)$ \\
\hline
\end{tabular}

Data from references $5,7,8,10,11,15,18$ were not derivable for lack of data, different methods of data collection or lack of defined elderly group data. Data are expressed as mean \pm SD [or median, IQR] and number and percentages. CPB, cardiopulmonary bypass; ACC, aortic cross clamp; DHCA, deep hypothermic circulatory arrest.

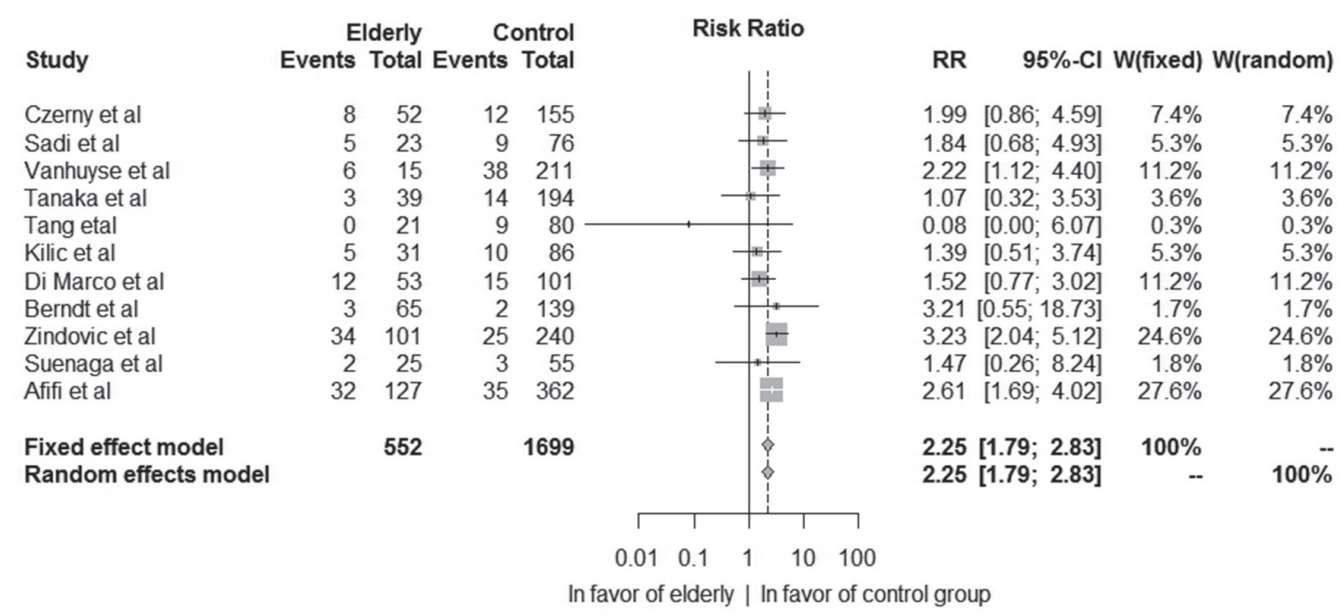

Figure 2 Forest plot of the pooled risk ratio for short-term mortality (test for overall effect $\mathrm{Z}=7.032, \mathrm{P}<0.001$; Heterogeneity test: $\mathrm{I}^{2}=0 \%$, $\left.\mathrm{Chi}^{2}=7.1, \mathrm{df}=10, \mathrm{P}=0.6271\right)$.

(25.3\%). A total of 110 events were reported in the elderly group with a pooled short term mortality of $19.9 \%$ : elderly patients had a significant higher risk for post-operative mortality compared to younger patients (pooled incidence $19.9 \%$ vs. $14.9 \%$; RR $=2.25 ; 95 \%$ CI, $1.79-2.82 ; \mathrm{I}^{2}=0 \%$; $\mathrm{P}<0.0001)$ (Figure 2). Four studies $(9,11,13,15)$ accounted for $74.7 \%$ of the entire analysis weight, but there was no significant heterogeneity between the studies. The overall pooled short term mortality for the elderly groups only, calculated in 17 studies, was $18.8 \%$ (Random effect model $16.7 \%$; $95 \%$ CI, $\left.12.06-22.72 ; \mathrm{I}^{2}=80.1 \%\right)$.

\section{Post-operative stroke}

Only seven studies $(1,6,7,11-14)$ reported post-operative stroke rates with an incidence in the elderly group ranging 


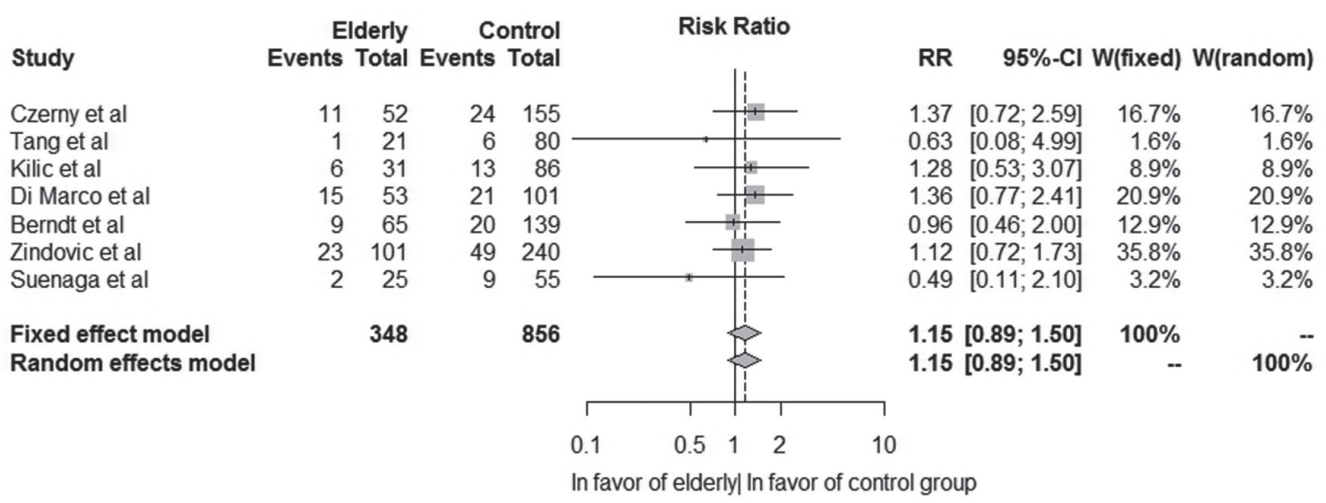

Figure 3 Forest plot of the pooled risk ratio for post-operative stroke rate (Test for overall effect $\mathrm{Z}=1.0648, \mathrm{P}=0.287$; Heterogeneity tests: $\left.\mathrm{I}^{2}=0 \%, \mathrm{Chi}^{2}=2.56, \mathrm{df}=5, \mathrm{P}=0.86\right)$.

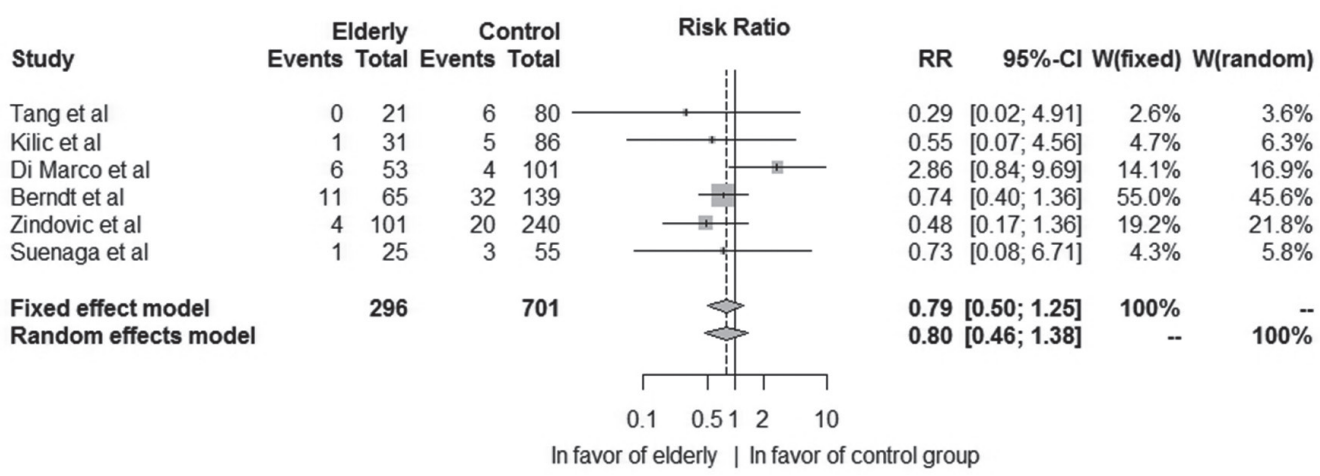

Figure 4 Forest plot for the pooled risk ratio for developing acute kidney injury (AKI) after surgery (Test for overall effect: Fixed effect model: $Z=-1.014, P=0.31$, Random effect model: $Z=-0.8053 ; P=0.42$; Heterogeneity tests: $\left.\mathrm{I}^{2}=14 \%, Q=5.82, d f=5, P=0.32\right)$.

from $4.76 \%$ to $28.3 \%$, with a pooled rate of $19.2 \%$. A total of 348 elderly patients over an overall population of 1,204 patients were included into this meta-analysis. Figure 3 describes the weighted meta-analysis for stroke incidence after surgery: there were no significant differences between the elderly and the young cohort with a RR 1.15 (95\% CI, 0.89-1.49; $\left.\mathrm{I}^{2}=0 \% ; \mathrm{P}=0.28\right)$.

\section{Post-operative acute kidney injury}

Post-operative AKI was evaluated in six studies (1,6,11-14) and involved 296 elderly patients in an overall population of 997 patients (29.7\%). The pooled incidence of postoperative AKI in elderly was $7.8 \%$. Figure 4 shows the results of meta-analysis for AKI incidence. There was no significant difference between the two groups, although a tendency towards a smaller rate of AKI was found in elderly patients $(\mathrm{RR}=0.79 ; 95 \% \mathrm{CI}, 0.50-1.25 ; \mathrm{P}=0.31$ in the fixed effect model and $\mathrm{RR}=0.80 ; 95 \% \mathrm{CI}, 0.46-1.38 ; \mathrm{P}=0.42$ in the random effect model.). The heterogeneity was higher in this analysis, although not statistically significant $\left(\mathrm{I}^{2}=14 \%\right.$; $\mathrm{Q}$ Test $\mathrm{P}$ value $=0.32$ ).

\section{Sub-analysis in octogenarians}

A further analysis was conducted to investigate the primary outcomes in those studies which had a cut-off age of 80 years. For short term mortality, the analysis was conducted on four studies $(6,7,9,14)$ : results were similar to the overall analysis with a RR of 2.30 (95\% CI, 1.29-4.09; P=0.0047). The heterogeneity was higher than the overall model, with $\mathrm{I}^{2}=23.2 \%$ and the pooled mortality rate for this subgroup of patients was $30.9 \%$ (random effect model $25.2 \%$; $95 \%$ CI, $\left.10.2-50 \% ; \mathrm{I}^{2}=70.8 \%\right)$. The sub-analysis for stroke involved three papers $(4,7,14)$ with a RR of $1.09(95 \%$ CI, 0.43-2.72; $\mathrm{P}=0.84$ ) for the fixed effect model and 1.05 
(95\% CI, 0.30-3.56, $\mathrm{P}=0.08$ ) for the random effect model. Similarly, in this sub-analysis of stroke, the heterogeneity was higher than the overall model $\left(\mathrm{I}^{2}=41.8 \%\right)$. The incidence of AKI was also included in the sub-analysis: in this case, the RR was 0.51 (95\% CI, 0.089-2.94, $\mathrm{P}=0.46$ ) for fixed and random effect model and $\mathrm{I}^{2}=0 \%$.

\section{Discussion}

Due to the continuously increasing life-expectancy in the western countries, a growing number of elderly patients are affected by $\mathrm{AAD}$ and frequently undergo complicated and very high risk surgical procedures to repair it. Without surgical intervention, about $75 \%$ of the patients die within two weeks of the onset of symptoms (18) and therefore the surgeon faces a therapeutic dilemma: to operate or not? Several studies have shown that surgery is preferable to the medical management $(4,18)$, but the results of surgical treatment in this subset of patients have been reported only in retrospective analysis or a small proportion of patients from international registries. A previous meta-analysis focused on octogenarians (4) have already demonstrated the feasibility of surgical repair of AAD in elderly patients. In their analysis, the authors supported a confident approach towards emergency repair in the elderly. Our results support this conclusion. In our analysis, although the early mortality rate was higher in the elderly group, with a pooled RR of 2.27 , postoperative stroke and AKI rate seemed not to be affected by age. This is in line with the recent report of the GERAADA (18). In their analysis involving 640 elderly patients ( $>70$ years) out of a population of 2,137 type A aortic dissections, the authors, while reporting a significant linear correlation between age and operative mortality risk, have found only a weak association between age and neurological events (18). The pooled mortality in our analysis was still very high (18.8\% for the overall studies). In the octogenarians though, the short-term mortality rate appeared to be better (30.9\%) compared to a previous meta-analysis on the same population of study (4). Low incidences of postoperative stroke have been also reported by other authors, although in small retrospectively analyzed samples $(11,14)$. Piccardo and colleagues (17), in their retrospective analysis of 79 octogenarians undergoing type A aortic dissection surgery, highlighted the importance of the clinical presentation as a factor affecting the surgical outcome and demonstrated that in uncomplicated $\mathrm{AAD}$, early and midterm outcomes are excellent and similar to those in published series of younger patients. Even in our analysis, the incidence of stroke appeared to be acceptable and not affected by age. In fact, the pooled analysis demonstrated a RR of 1.15 with a $95 \%$ confidence intervals of $0.89-1.50(\mathrm{P}=0.28)$. The definitions of stroke were not univocal between the studies, but the incidences appeared to be similar in the two groups, ranging between $5 \%$ and $28.3 \%$ in the elderly group and between $7.5 \%$ and $20.7 \%$ in the younger groups.

Similar results have been found with the analysis of the AKI. In this case, the pooled analysis showed results in favor of the elderly population, although they were not significant. The incidence of this complication was between 0 and $16 \%$ in the elderly group and between $3.9 \%$ and $23.02 \%$ in the young group: in this case, AKI seems to be more frequent in the younger population, although this difference was not significant.

In terms of surgical procedures performed, there is a tendency toward a less aggressive surgical approach in elderly patients as it is considered to be safer and more effective than complex surgery (18). This has been clearly showed with the GERAADA registry where there was a correlation between age and type of surgical procedures performed, with a higher number of supra-coronary aortic replacement, in the elderly and a higher number of infracoronary replacements in young patients. A less aggressive surgical approach seems to be favorable in elderly patients and for some authors is a key factor in reducing reducing operative time and mortality (17). In consideration of the short life expectancy, especially in octogenarians, priority should be given to prevent aortic rupture and complete the repair of the acute dissection. In this sense, one of the main consideration to make is regarding the life expectancy after complicated surgical repairs. Interestingly, the survival rate in the papers considered in the meta-analysis was acceptable, ranging from $32 \%$ to $100 \%$ at 5 years. Moreover, Suenaga and colleagues (14) have found no significant difference between the survival rate of octogenarians undergoing type A dissection surgery and the overall octogenarians population in the same area, supporting the idea that if the patients survive to the immediate post-operative outcome, the life expectancy could be similar to the general agematched population. Even the quality of life after surgery appears to be reasonable as shown by Tang and colleagues (6). In their study, focused on octogenarians, the authors found good results in terms of emotional and social well-being, albeit with slightly worse physical functioning. They have also reported exceptional short-term results, with an in-hospital mortality rate of $0 \%$ and $100 \%$ survival 
rate at five years (6). The authors have attributed their very good results to their protocol of approach that avoids delays in transfer, transport and diagnosis confirmation, allowing precarious patients to be supported on cardio-pulmonary bypass as quick as possible.

The main limitation of this meta-analysis is the presence of only retrospective studies with small sample size and wide-ranging long-term survival results. Another limitation could be represented by an intrinsic selection bias. Most surgeons would operate almost all the young patients with $\mathrm{AAD}$, although they would be more selective when the patient is elder. It is complicated to verify this assumption, as no clear data are obtainable regarding the reasons for refusing surgery in this sub-group of patients.

In conclusion, $\mathrm{AAD}$ repair in elderly, whilst associated with a higher surgical mortality rate, can be performed with acceptable short-term outcomes and reasonable mid-term survival rates.

\section{Acknowledgements}

None.

\section{Footnote}

Conflicts of Interest: The authors have no conflicts of interest to declare.

\section{References}

1. Kilic A, Tang R, Whitson BA, et al. Outcomes in the current surgical era following operative repair of acute Type A aortic dissection in the elderly: a singleinstitutional experience. Interact Cardiovasc Thorac Surg 2013;17:104-9.

2. Komatsu K, Takano T, Terasaki T, et al. Surgical outcomes of acute type A aortic dissection in elderly patients. Ann Thorac Surg 2014;97:1576-81.

3. Rampoldi V, Trimarchi S, Eagle KA, et al. Simple risk models to predict surgical mortality in acute type A aortic dissection: the International Registry of Acute Aortic Dissection score. Ann Thorac Surg 2007;83:55-61.

4. Biancari F, Vasques F, Benenati V, et al. Contemporary results after surgical repair of type A aortic dissection in patients aged 80 years and older: a systematic review and meta-analysis. Eur J Cardiothorac Surg 2011;40:1058-63.

5. Rylski B, Suedkamp M, Beyersdorf F, et al. Outcome after surgery for acute aortic dissection type $\mathrm{A}$ in patients over 70 years: data analysis from the German Registry for Acute Aortic Dissection Type A (GERAADA). Eur J Cardiothorac Surg 2011;40:435-40.

6. Tang GH, Malekan R, Yu CJ, et al. Surgery for acute type A aortic dissection in octogenarians is justified. J Thorac Cardiovasc Surg 2013;145:S186-90.

7. Czerny M, Krähenbühl E, Reineke D, et al. Mortality and neurologic injury after surgical repair with hypothermic circulatory arrest in acute and chronic proximal thoracic aortic pathology: effect of age on outcome. Circulation 2011;124:1407-13.

8. Sadi L, Tønnessen T, Pillgram-Larsen J. Short and long-term survival in type A aortic dissection justifies the operative risk and effort. Scand Cardiovasc J 2012;46:45-50.

9. Vanhuyse F, Maureira P, Laurent N, et al. Surgery for acute type A aortic dissection in octogenarians. J Card Surg 2012;27:65-9.

10. Tanaka M, Kimura N, Yamaguchi A, et al. In-hospital and long-term results of surgery for acute type A aortic dissection: 243 consecutive patients. Ann Thorac Cardiovasc Surg 2012;18:18-23.

11. Di Marco L, Pacini D, Leone A, et al. Long-term outcome after acute type A aortic dissection: does an age limit still exist? J Cardiovasc Surg (Torino) 2014;5 5:359-65.

12. Berndt R, Haneya A, Jussli-Melchers J, et al. Outcome after surgery for acute aortic dissection type $\mathrm{A}$ in the elderly: a single-center experience. Thorac Cardiovasc Surg 2015;63:113-9.

13. Zindovic I, Sjögren J, Bjursten H, et al. Impact of Hemodynamic Instability and Organ Malperfusion in Elderly Surgical Patients Treated for Acute Type A Aortic Dissection. J Card Surg 2015;30:822-9.

14. Suenaga E, Sato M, Fumoto H. Ascending aortic replacement for acute type A aortic dissection in octogenarians. Gen Thorac Cardiovasc Surg 2016;64:138-43.

15. Afifi RO, Sandhu HK, Leake SS, et al. Determinants of Operative Mortality in Patients With Ruptured Acute Type A Aortic Dissection. Ann Thorac Surg 2016;101:64-71.

16. Zheng J, Lu S, Sun X, et al. Surgical management for acute type A aortic dissection in patients over 70 years-old. J Cardiothorac Surg 2013;8:78.

17. Piccardo A, Le Guyader A, Regesta T, et al. Octogenarians with uncomplicated acute type a aortic dissection benefit from emergency operation. Ann 
Thorac Surg 2013;96:851-6.

18. Rylski B, Hoffmann I, Beyersdorf F, et al. Acute aortic dissection type A: age-related management and outcomes reported in the German Registry for Acute Aortic Dissection Type A (GERAADA) of over 2000 patients. Ann Surg 2014;259:598-604.

19. Matsushita A, Tabata M, Fukui T, et al. Outcomes of

Cite this article as: Bruno VD, Chivasso P, Guida G, Vohra HA. Surgical repair of Stanford type A aortic dissection in elderly patients: a contemporary systematic review and metaanalysis. Ann Cardiothorac Surg 2016;5(4):257-264. doi: 10.21037/acs.2016.06.03 contemporary emergency open surgery for type A acute aortic dissection in elderly patients. J Thorac Cardiovasc Surg 2014;147:290-4.

20. Malvindi PG, Modi A, Miskolczi S, et al. Acute type A aortic dissection repair in elderly patients. Eur J Cardiothorac Surg 2015;48:664-70; discussion 671. 